\title{
Fast 4-nitrophenol Reduction Using Gelatin Hydrogel Containing Silver Nanoparticles
}

\author{
Mazhar Ul-Islam, ${ }^{1^{*}}$ Jawad Ali, ${ }^{2}$ Waliullah Khan, ${ }^{3}$ Adnan Haider, ${ }^{4}$ Nasrullah Shah, ${ }^{3}$ Md. Wasi Ahmad, ${ }^{1}$ Muhammad Wajid Ullah ${ }^{5 *}$ and \\ Guang Yang ${ }^{*}$
}

The current study reports the synthesis of gelatin hydrogel containing silver nanoparticles (AgNPs) and its role as a catalyst. A porous gelatin hydrogel, with pore size of 15-100 nm, was prepared by cooling its hot solution to room temperature with glutaraldehyde as crosslinker. The gelatin hydrogel was immersed in $1 \mathrm{mM}$ silver nitrate solution for $24 \mathrm{~h}$ through ex-situ impregnation. The impregnation of AgNPs was confirmed through field-emission scanning electron microscopy (FE-SEM) which showed uniform dispersion of nanoparticles on the hydrogel surface and partial impregnation into the matrix. Thermogravimetric analysis (TGA) showed the impregnation of $9 \mathrm{wt} \%$ of AgNPs in the hydrogel composite which also contributed to its improved thermal properties. X-ray diffraction (XRD) analysis confirmed the amorphous nature of gelatin and addition of AgNPs. The AgNPs/gelatin hydrogel effectively reduced 4-nitrophenol by sodium tetrahydroborate at a reaction rate constant of 0.498 $\min ^{-1}$. The prepared AgNPs/gelatin hydrogel could be used as a catalyst for other similar type of reactions.

Keywords: Gelatin; Hydrogel; Silver nanoparticles; Catalyst

Received 25 February 2019, Accepted 3 April 2019

DOI: $10.30919 / \mathrm{es} 8 \mathrm{~d} 504$

\section{Introduction}

Gelatin is known to mankind since the $16^{\text {th }}$ century. At present, it is used for different applications in pharmaceutics, food, biomedical, and photographic industries; therefore, it is produced at large-scale. The general chemical structure of gelatin is given in scheme 1. Its chemical structure contains numerous $-\mathrm{NH}_{2}, \mathrm{NH}$, and other oxygen containing functional groups which interact with different metal ions and nanoparticles for diverse functional applications. ${ }^{1-3}$ Gelatin is mostly used in the form of a hydrogel which is a swollen crosslinked polymer chain network. Its hydrogels can accommodate as high as $95 \%$ of water by weight. Several studies have reported the use of hydrogels or hydrogel-forming polymer coatings such as chitosan, bacterial cellulose, and carboxymethyl cellulose on other substrate for the use of hosting the catalytic metal nanoparticles. ${ }^{48}$ In addition, the gelatin hydrogels have also been explored for the adsorption and biocompatibility studies. ${ }^{9-11}$

'Department of Chemical Engineering, College of Engineering, Dhofar University, Salalah, Sultanate of Oman

${ }^{2}$ Department of Environmental Science, Faculty of Life Sciences and Informatics, Balochistan University of Information Technology Engineering and Management Sciences, Quetta, Pakistan

${ }^{3}$ Department of Chemistry, Abdul Wali Khan University Mardan, Mardan, Pakistan

${ }^{4}$ Department of Chemistry, Kohat University of Science and Technology, Kohat, Khyber Pakhtunkhwa, Pakistan

${ }^{5}$ Department of Biomedical Engineering, Huazhong University of Science and Technology, Wuhan 430074, China

*E-mail:mulislam@du.edu.om; wajid_kundi@hust.edu.cn;

yang_sunny@yahoo.com
Metals and their oxide nanoparticles are currently attaining increasing interest ${ }^{12}$ owing to their extensive use in different fields such as for optical, sensor, and biomedical applications due to their catalytic, antibacterial, and antifungal properties. ${ }^{13-18}$ Their use as catalysts is one of the most important properties of nanoparticles. ${ }^{6,13,19,20}$ Owing to their large exposed surface area as compared to their bulk form, high reaction rates can be achieved by using only a small amount of nanoparticles. ${ }^{15,21}$ Besides their known high catalytic activity, the nanoparticles tend to aggregate during repeated use due to their high surface energy which eventually lowers their activity. To overcome this issue, researchers have developed different approaches to prevent their aggregation such as their stabilization by using different organic compounds, block copolymers, and using biological substrates as support. ${ }^{22,23}$ A vast literature is available on use of different metal and their oxides-based nanoparticles ${ }^{3,24-27}$ for various applications; however, the potential of silver nanoparticles (AgNPs) is rarely explored for their environmental applications as reducing agents for organic wastes. The silver nanoparticles are expected to possess high catalytic properties; however, these also encounter the issue of aggregation. Some researchers have used different synthetic hydrogels to overcome their aggregation, ${ }^{22,28}$ however, such synthetic hydrogels increase the overall cost of specific environmental application based on such Ag-based hydrogels. ${ }^{29-31}$ Therefore, the current study was aimed to use gelatin; an environment-friendly, biodegradable, and a low-cost substrate, as a support material for silver nanoparticles to explore its catalytic performance.

Currently, wastewater purification has gained increasing interest because the daily activities performed by industries are contaminating the adjacent water bodies ${ }^{7,32}$ which directly affects the living organism dependent on those water bodies. For instance, textile industries discharge toxic reactive dyes to the nearby streams. Similarly, 
pharmaceutical industries discharge toxic organic compounds to the water streams such as aromatic compounds. These compounds not only affect the aquatic life but also impose adverse effects on locality. Therefore, their removal is of prime important as suggested by environmentalists. Among the different techniques used for their removal, the catalytic transformation of these compounds to less-toxic compounds are one of the possible solutions to this problem. To lower the cost of the catalytic process, it is highly desired to recover the catalyst and re-use it in another batch.

The current study was aimed to fabricate silver nanoparticles impregnated gelatin hydrogel through cross-linking of glutaraldehyde for application in catalysis for reduction of 4-nitrophenol by sodium tetrahydroborate. This fabricated gelatin/silver nanocomposite was evaluated for its performance by catalyzing the 4-nitrophenol (4-NP) reduction to 4-aminophenol (4-NP) in its aqueous solution state. The developed material can find potential applications as a catalyst in degrading different organic material from industrial wastes.

\section{Experimental section}

\subsection{Materials}

The different chemical reagents used in the current study including gelatin powder, sodium tetrahydroborate, silver nitrate, and 4nitrophenol were purchased from a reputable chemical supplier and were used without further processing. Deionized and distilled water was used in all experiments.

\subsection{Preparation of gelatin hydrogels}

The two different hydrogels of gelatin were prepared in deionized distilled water. Briefly, a $10 \mathrm{wt} \%$ gelatin was dissolved in distilled water by heating at $60^{\circ} \mathrm{C}$ with continuous stirring. After complete dissolution, an appropriate amount of glutaraldehyde solution was added and stirred for $10 \mathrm{~min}$. The solution was cooled to room temperature, which ultimately changed into a hydrogel. This hydrogel was used in the catalytic studies. A similar procedure was adopted for preparation of $3 \mathrm{wt} \%$ solution. This hydrogel was used for imaging of nanoparticles.

\subsection{Preparation of silver nanoparticles synthesis and immersion in gelatin hydrogel}

A chemical reduction method was used for synthesis of silver nanoparticles in gelatin hydrogel. For preparation of silver nanoparticles in the gelatin hydrogel, a $1 \mathrm{mM}$ silver nitrate solution was used. Typically, $5 \mathrm{~g}$ of hydrogel prepared by the above stated method was immersed in a $50 \mathrm{~mL}$ silver nitrate solution for $5 \mathrm{~h}$. After silver ions absorption, the hydrogel was put inside a freshly prepared $15 \mathrm{mM}$ sodium tetrahydroborate solution. The hydrogel turned dark brown immediately after immersion in reducing agent solution. This hydrogel was repeatedly washed with deionized distilled water to remove the un-reacted silver ion and nanoparticles on its surface.

\subsection{Characterization}

The morphological features of the freeze-dried gelatin hydrogel and the presence of silver nanoparticles in its matrix were observed through field emission scanning electron microscope (Hitachi S-4800 \&EDX350 (Horiba) FE-SEM (Tokyo Japan). Prior to observation via FE$\mathrm{SEM}$, the samples were coated with osmic acid $\left(\mathrm{OsO}_{4}\right)$ with VD HPCISW osmium coater (Tokyo Japan). XRD analysis was carried out using X-ray diffractometer (X'Pert-APD Philips, Netherlands) with an $\mathrm{X}$-ray generator $(3 \mathrm{~kW})$ and anode $(\mathrm{LFF} \mathrm{Cu})$. The radiation was $\mathrm{CuK}-\alpha$ at $1.54 \AA$, the $\mathrm{X}$-ray generator tension and current was $40 \mathrm{kV}$ and 30 $\mathrm{mA}$, respectively, and the angle of scanning varied from 20 to $80^{\circ}$. The thermogravimetric analysis (TGA) was performed using a thermogravimetric/differential thermal analyzer (Seiko Instruments Inc.). Typically, 5 to $10 \mathrm{mg}$ sample (freeze-dried) was used in the experiment. The sample was heated at a heating rate of $10^{\circ} \mathrm{C}$ per min in a sealed ceramic crucible sample holder up to $800^{\circ} \mathrm{C}$ temperature in the inert atmosphere. For the catalytic experiments, a UV-visible spectrophotometer (Thermo Scientific) was used. For analysis, the solution in a quartz cuvette was placed in the sample holder with a reference cuvette containing water, for measurements.

\subsection{Catalyst testing in a model reduction reaction}

(a)
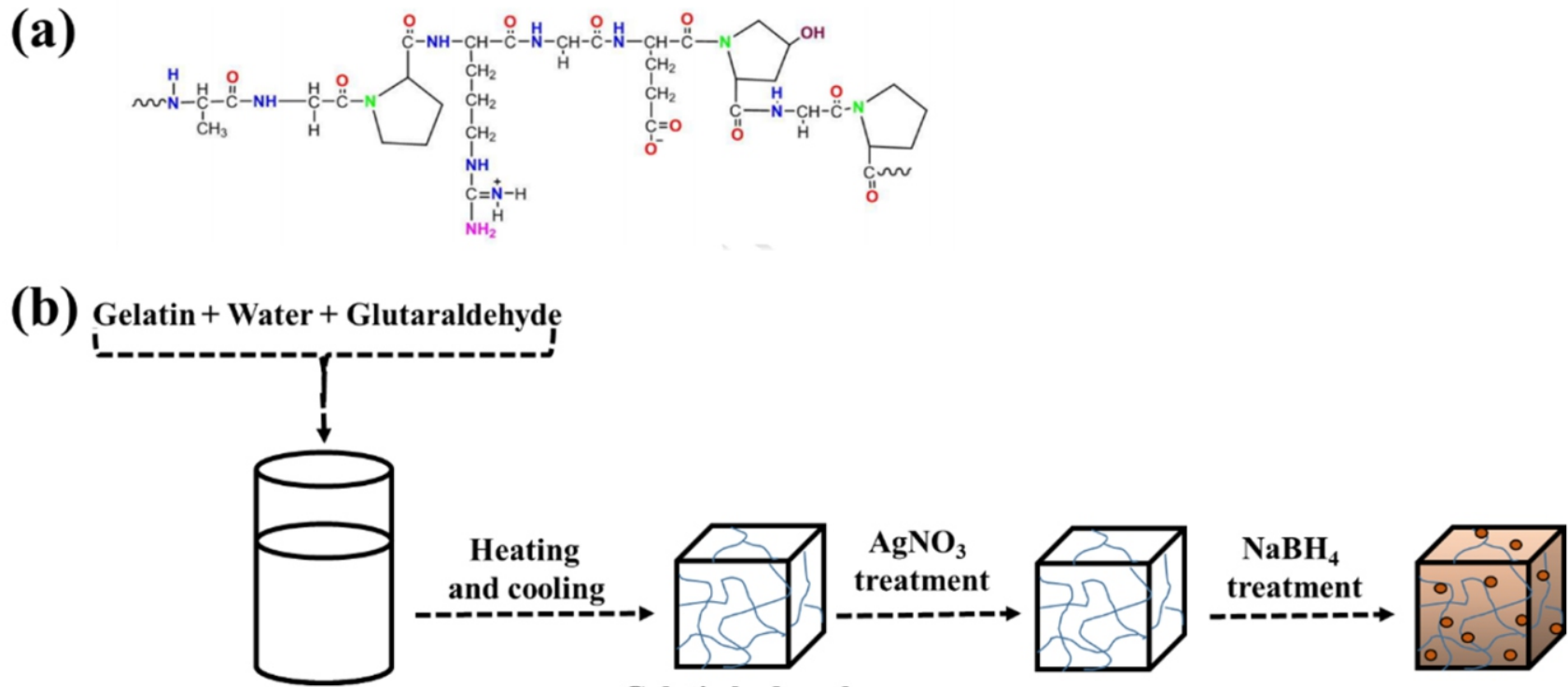

Gelatin hydrogel

Scheme 1 Illustration of (a) chemical structure of gelatin (b) formation of its hydrogel containing impregnated Ag NPs and their preparation process. 
To investigate the role of gelatin hydrogel containing Ag nanoparticles as a catalyst, it was meshed into small pieces (below $1 \mathrm{~mm}$ ) by repeatedly applying and releasing the thumb pressure over it. A 4nitrophenol (4-NP) in deionized distilled water stock solution was prepared with a concentration of $1.4 \times 10^{-4} \mathrm{M}$. Thereafter, $40 \mathrm{~mL}$ of 4NP solution was taken in a beaker containing $0.35 \mathrm{~g}$ of gelatin hydrogel supported Ag nanoparticles and stilled for $3 \mathrm{~min}$. The reduction reaction was initiated by adding $0.5 \mathrm{~g}$ sodium tetrahydroborate into the beaker. At this point, approximately $3 \mathrm{~mL}$ solution from beaker was subjected to UV-visible absorption spectroscopy measurements at constant time interval.

\section{Results and discussion}

The chemical structure of gelatin and $\mathrm{Ag}$ nanoparticles impregnated gelatin fibers are illustrated in scheme 1. The dissolution of two different concentrations of gelatin in water revealed that gelatin with lower concentration was readily dissolved in hot water at $60{ }^{\circ} \mathrm{C}$ and formed a clear solution. The addition of crosslinker facilitated the gel formation process and the solution turned into a hydrogel upon cooling to room temperature. The $\mathrm{Ag}$ nanoparticles were incorporated into the hydrogel through ex-situ impregnation where the hydrogel absorbed silver ions from silver nitrate solution alongside their synthesis process. Further treatment of hydrogel with sodium tetrahydroborate reduced the absorbed ions to Ag NPs in the gelatin hydrogel. The Ag NPs attached to gelatin fibers on the surface as well as into the matrix.

\subsection{Morphological features of AgNPs/gelatin hydrogel}

The structural morphology of pure gelatin and AgNPs containing gelatin hydrogel were observed through FE-SEM. Fig. 1a shows the low magnification FE-SEM image of the vacuum-dried pure gelatin hydrogel prepared from a $3 \mathrm{wt} \%$ solution. It shows the typical porous morphology of hydrogels as reported in earlier research works. ${ }^{10,11}$ The pore size of gelatin hydrogel was in the range of 15-100 nm. Such a large dimension pores effectively allow the free solvent exchange with inner area of the hydrogel. Therefore, it can support the nanoparticles for their application in catalysis. Fig. 1b shows the highly magnified FE-SEM image of the vacuum-dried pure gelatin hydrogel. The observed morphological view indicate that the surface of the hydrogel was smooth. Fig. 1c shows the highly magnified FE-SEM image of AgNPs containing gelatin hydrogel prepared from a $3 \mathrm{wt} \%$ solution. This image shows the bright spots of AgNPs as shown by the red arrows. It could be seen that NPs are dispersed on the surface and probably impregnated inside the gelatin matrix. It is expected that the attached NPs will bestow gelatin with new features. The size of the nanoparticles was in the range of 100-500 $\mathrm{nm}$. Indeed, the actual particle size of AgNPs is much smaller as indicated in XRD analysis; however, the relatively higher particle size observed here may be due to the particle aggregation.

\subsection{Thermogravimetric and X-Ray diffraction analysis of AgNPs/gelatin hydrogel}

Fig. 2 shows the TGA curves of freeze-dried pure gelatin and AgNPs/gelatin hydrogel samples. The TGA curve showed threedifferent weight loss regions. The initial weight loss ranging about 7 to $8 \%$ in both samples could be attributed to the loss of water molecules. After that, a strong weight loss in the temperature window of the 220 ${ }^{\circ} \mathrm{C}$ to $400{ }^{\circ} \mathrm{C}$ was observed in pure gelatin sample which could be attributed to the degradation of gelatin polymer chains in the sample.
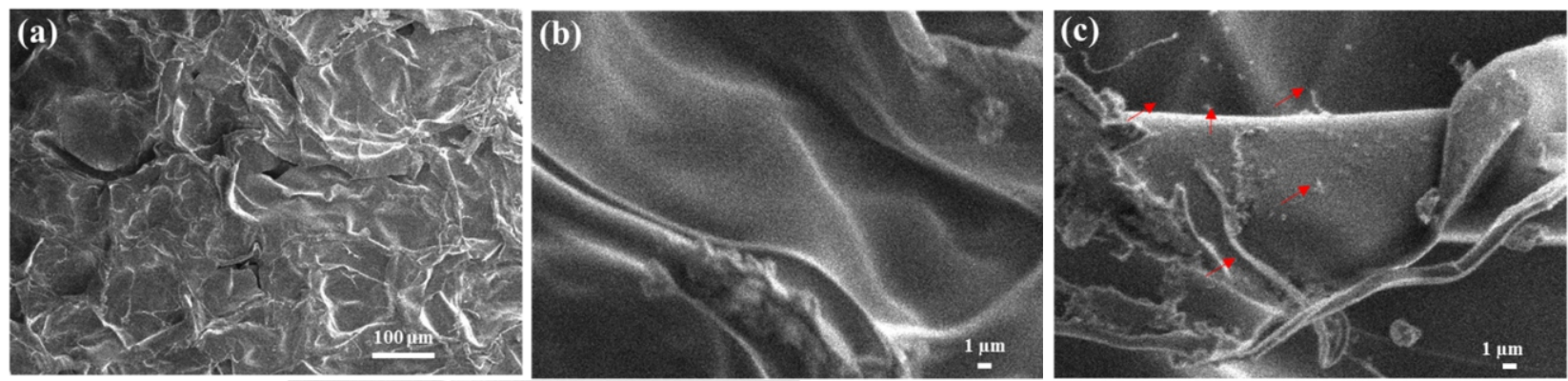

Fig. 1 FE-SEM images of the prepared hydrogel material: (a) pure hydrogel at low and (b) at high magnifications, and (c) AgNPs impregnated gelatin hydrogel.

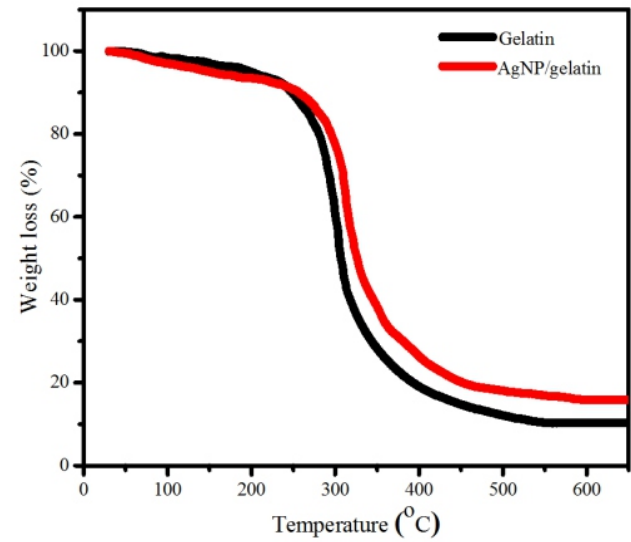

Fig. 2 TGA thermograms of pure gelatin and AgNPs/gelatin hydrogels.

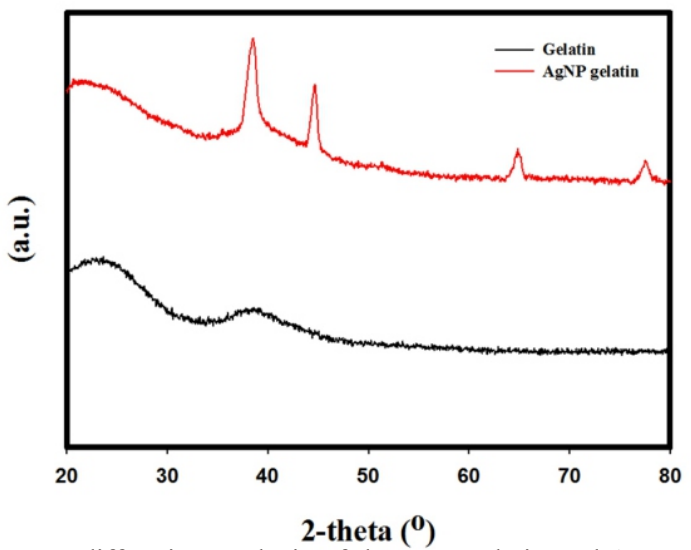

Fig. 3 X-ray diffraction analysis of the pure gelatin and AgNPs/gelatin hydrogels. 
After $455^{\circ} \mathrm{C}$, there was no change in the weight and final residue of $10 \%$ was obtained. In the AgNPs/gelatin sample, the major weight loss started at $260{ }^{\circ} \mathrm{C}$ and continued until a constant weight of $19 \%$ was obtained. The difference between the weights of gelatin and AgNPs/gelatin was about 9\%. These observations demonstrate that the embedded nanoparticles slightly enhanced the thermal stability of hydrogel. Furthermore, the TGA analysis provided the evidence of presence of $9 \mathrm{wt} \%$ of AgNPs in the composite hydrogel. The TGA results are the approximate for amount of $\mathrm{Ag}$ nanoparticles in the hydrogel. Similar method for the approximation of the metal nanoparticles was reported by several other studies. ${ }^{4,1533}$

Fig. 3 represents the XRD patterns of the freeze-dried gelatin and AgNPs/gelatin hydrogel samples. Gelatin is a known amorphous polymer; therefore, we did not observe any diffraction peak in the XRD curve of the gelatin except an amorphous halo at 2-theta of $20^{\circ}$. In contrast, the XRD curve of the AgNPs/gelatin showed several diffraction peaks which could be attributed to the presence of $\mathrm{Ag}$ nanoparticles in the hydrogel with cubic phase crystal structure as reported previously ${ }^{15}$. The size of the nanoparticles was calculated by the Scherrer equation which was estimated to be $22 \mathrm{~nm}$.

\subsection{Catalytic properties of AgNPs/gelatin hydrogel}

The catalytic properties of AgNPs-loaded gelatin hydrogel were determined by using a model reduction reaction of 4-NP by sodium tetrahydroborate. 4-NP finds wide applications in numerous industries. However, it is considered as priority pollutants by the US Environmental Protection Agency because it is found to be responsible for liver-related problems, kidney damage, feverness, and headache on inhalation or ingestion. Therefore, it is very important to reduce and convert it into less toxic amino derivative. During the reduction process, the yellow color of 4-NP solution slowly turns colorless upon introduction of AgNPs containing gelatin hydrogel. This color change could be detected by spectrophotometer which measures the absorption spectra. Fig. 4 shows the time-dependent UV-visible absorption spectra

Table 1 Comparison of the AgNPs in gelatin hydrogel catalytic efficiency with other catalysts for the reduction of 4-NP.

\begin{tabular}{|c|c|c|c|}
\hline Catalyst & Reaction time (s) & $K_{a p p}\left(\mathbf{s}^{-1}\right)$ & Ref. \\
\hline (p(AMPS) -Co hydrogel & 1680 & $2.0 \times 10^{-3}$ & 34 \\
\hline Au doped meso -porous Boehmite film & 1920 & $1.7 \times 10^{-3}$ & 35 \\
\hline $\mathrm{Al}_{2} \mathrm{O}_{3} @ \mathrm{Ag}_{7,8} \mathrm{QCs}$ & 1080 & $8.23 \times 10^{-3}$ & 36 \\
\hline Sheet-like $\left(\mathrm{Co}_{0.376} \mathrm{Ni}_{0.62}\right)_{0.85} \mathrm{Se}$ & 840 & $3.5 \times 10^{-3}$ & 37 \\
\hline Pd-SPB & 1200 & $4.41 \times 10^{-3}$ & 38 \\
\hline Au@HSNs_C & 1800 & $1.0 \times 10^{-3}$ & 39 \\
\hline $\mathrm{Fe}_{3} \mathrm{O}_{4} @ \mathrm{CTS}-\mathrm{Au} \mathrm{NPs}(\mathrm{A})$ & 1320 & $8.6 \times 10^{-3}$ & 40 \\
\hline Hollow capsule -stabilized AuNPs & 1800 & $2.0 \times 10^{-3}$ & 41 \\
\hline AgNPs on gelatin hydrogel & 840 & $8.22 \times 10^{-3}$ & This work \\
\hline
\end{tabular}
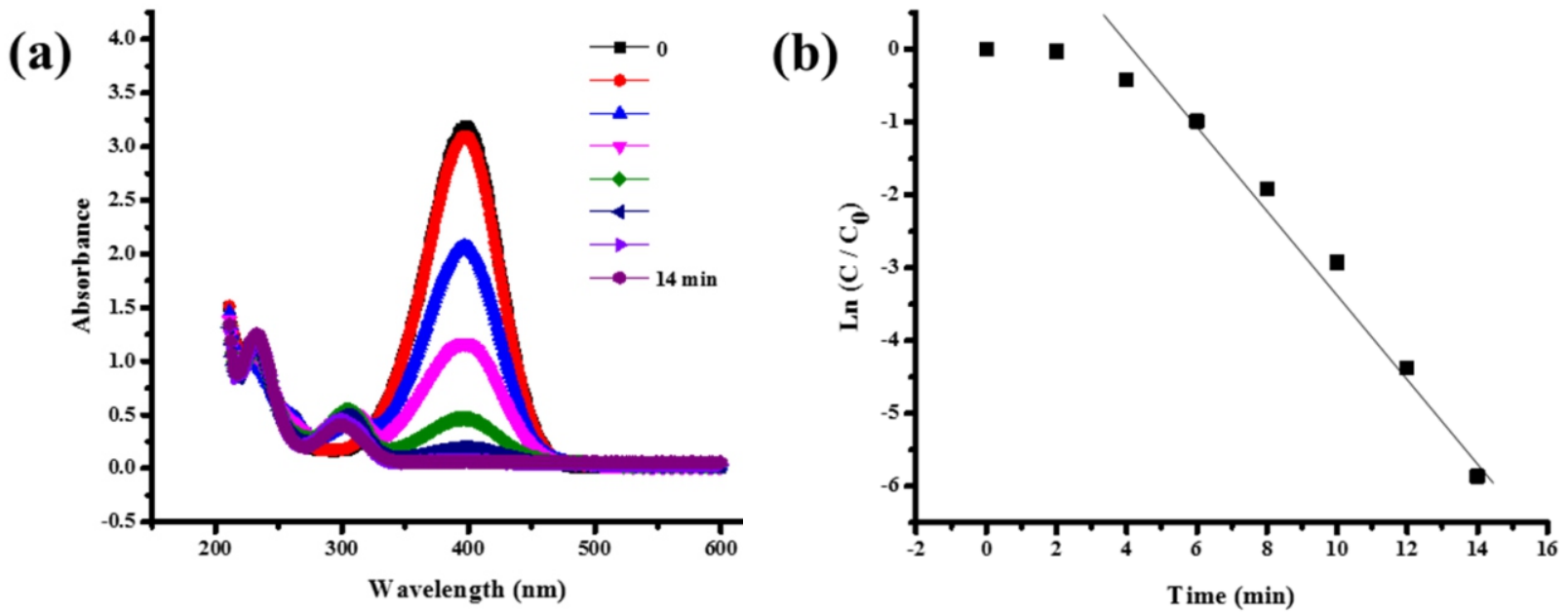

Fig. 4 Time-dependent spectral changes in 4-NP solution during its reduction by sodium tetrahydroborate in the presence of AgNPs loaded gelatin hydrogel (a) and corresponding pseudo-first order kinetic plot (b). 
measured from 4-NP solution consisted of AgNPs containing hydrogel and $\mathrm{NaBH}_{4}$. In this experiment, $0.7 \mathrm{~g}$ of sodium tetrahydroborate was directly introduced to $0.08 \mathrm{mM}$ 4-NP solution $(50 \mathrm{ml})$. It was observed that the main peak located at $400 \mathrm{~nm}$ was gradually reduced and reached to its minimum in $40 \mathrm{~min}$, indicating $98 \%$ reduction of this peak. At the same time, a new peak slowly evolved at $302 \mathrm{~nm}$. Based on the available literature, such observations are the indication of successful transformation of 4-NP to 4-AP. The data from Fig. 4a was treated according to the equation of the pseudo-first-order kinetic reaction. It was plotted in the form of $\ln \left(\mathrm{C} / \mathrm{C}_{0}\right)$ values against time as shown in Fig. 4b. In Fig. $4 b$, the $C$ in $\ln \left(C / C_{0}\right)$ represents the concentration of 4-NP at given interval while $\mathrm{C}_{0}$ denotes the initial concentration. The calculated rate constant $\left(K_{\text {app }}\right)$ was $0.498 \mathrm{~min}^{-1}(8.22$ $\left.\times 10^{-3} \mathrm{~s}^{-1}\right)$. To check the stability, the AgNPs in gelatin hydrogel was used in repeated 4-NP to 4-AP transformation reaction. For each test, the AgNPs loaded hydrogel particles were easily separated by just a filtration process. Filtration process could not be used for the unsupported colloidal type nanoparticles. Table 1 compares the catalytic efficiency of catalyst (AgNPs/gelatin) prepared in the current study with those reported earlier. It is evident that AgNPs loaded gelatin hydrogel showed better performance for the same reduction reaction. Scheme 2 shows the conversion of the 4-NP to 4-AP in percentage against times use. For four times repeated use of the same catalytic AgNPs loaded hydrogel, the conversion of more than $80 \%$ of the 4-NP to 4-AP was achieved. Such results suggest that it could be used effectively for catalyzing other similar type of reduction chemical reactions.

\subsection{Mechanism}

The observed values of high rate constant and conversion (\%) could be attributed to the size and distribution of AgNPs. Literature studies revealed that the catalytic actives of nanoparticle are positively affected by reduction in the particle size. As a matter of fact, that the small sized nanoparticle exhibits very high catalytic activities. As per the XRD results the nanoparticle size was $22 \mathrm{~nm}$ whereas the nanoparticles were widely distributed in the gelatin hydrogel. The reduction of nitrobenzene was carried out with the help of $\mathrm{NaBH}_{4}$ and it is briefly summarized in Scheme 2.

\section{Conclusions}

In summary, we successfully synthesized AgNPs loaded hydrogels.
The hydrogel matrix contained AgNPs as evidenced by the XRD and SEM analyses. The prepared AgNPs loaded gelatin hydrogel was used as a reactor for water-based reactor where the nanoparticles played the role of a catalyst. By utilizing the prepared material in a typical 4 nitrophenol reduction reaction by sodium tetrahydroborate, a rate constant of $0.498 \mathrm{~min}^{-1}$ was observed. The material was easily recovered from one reaction and directly used for another reaction which showed good recyclability. The prepared AgNPs gelatin hydrogel could be used as a catalyst for other similar type of reactions.

\section{Conflict of interest}

There are no conflicts to declare.

\section{Acknowledgements}

This research was supported by TRC (FURAP /DU/18/010) Oman, National Natural Science Foundation of China (31270150, 51603079, 21774039), China Postdoctoral Science Foundation (2016M602291), and Fundamental Research Funds for Central Universities, Open Research Fund of State Key Laboratory of Polymer Physics and Chemistry, Changchun Institute of Applied Chemistry, Chinese Academy of Sciences.

\section{References}

1. S. B. Khan, M. S. Ahmed, T. Kamal, S. A. Khan, Y. Anwar, M. T. Saeed, A. M. Asiri, Curr. Nanosci., 2016, 12, 569-575.

2. T. Kamal, S. B. Khan, S. Haider, Y. G. Alghamdi and A. M. Asiri, Int. J. Biol. Macromol., 2017, 104, 56-62.

3. S. A. Khan, S. B. Khan, T. Kamal, M. Yasir and A. M. Asiri, Int. J. Biol. Macromol., 2016, 91, 744-751.

4. I. Ahmad, T. Kamal, S. B. Khan and A. M. Asiri, Cellulose, 2016, 23, $3577-$ 3588.

5. F. Ali, S. B. Khan, T. Kamal, Y. Anwar, K. A. Alamry and A. M. Asiri, Carbohydr. Polym., 2017, 173, 676-689.

6. S. Haider, T. Kamal, S. B. Khan, M. Omer, A. Haider, F. U. Khan and A. M. Asiri, Appl. Surf. Sci., 2016, 387, 1154-1161.

7. T. Kamal, N. Ali, A. Naseem, S. Khan and A. Asiri, Recent Pat. Nanotechnol., 2016, 10, 189-201.

8. Z. Shi, X. Gao, M. W. Ullah, S. Li, Q. Wang and G. Yang, Biomaterials, 2016, 111, 40-54

9. K. Ofokansi, G. Winter, G. Fricker and C. Coester, Eur. J. Pharm. Biopharm., 2010, 76, 1-9.

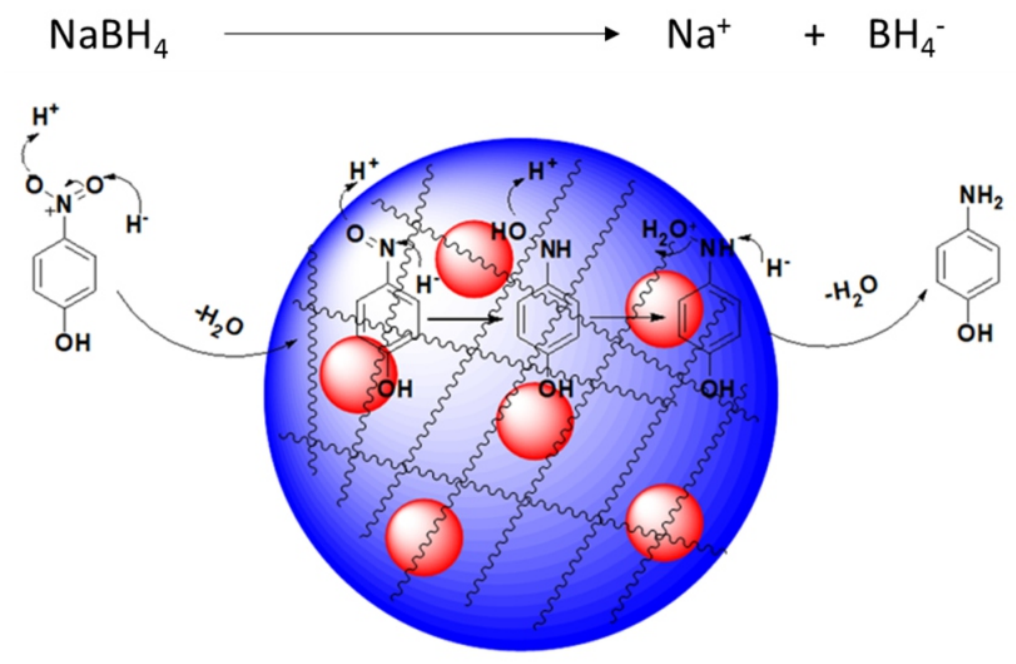

Scheme 2 Mechanism of 4-NP to 4-AP reduction using AgNPs containing gelatin hydrogel. 
10.S. Khan, M. Ul-Islam, M. Ikram, S. U. Islam, M. W. Ullah, M. Israr, J. H. Jang, S. Yoon and J. K. Park, Int. J. Biol. Macromol., 2018, 117, $1200-1210$.

11.S. Khan, M. Ul-Islam, M. Ikram, M. W. Ullah, M. Israr, F. Subhan, Y. Kim, J. H. Jang, S. Yoon and J. K. Park, RSC Adv., 2016, 6, 110840-110849.

12.T. Kamal, I. Ahmad, S.B. Khan, A.M. Asiri, Desalin. Water Treat., 2018, 136, 190-198.

13.V. Kumar and S. K. Yadav, J. Chem. Technol. Biotechnol., 2009, 84, 151157.

14.T. Kamal, J. Alloys Compd., 2017, 729, 1058-1063.

15.T. Kamal, I. Ahmad, S. B. Khan and A. M. Asiri, Carbohydr. Polym., 2017, 157, 294-302.

16.T. Kamal, Y. Anwar, S. B. Khan, M. T. S. Chani and A. M. Asiri, Carbohydr. Polym., 2016, 148, 153-160.

17.T. Kamal, M. Ul-Islam, S. B. Khan and A. M. Asiri, Int. J. Biol. Macromol., 2015, 81, 584-590.

18.M. W. Ullah, M. Ul-Islam, S. Khan, Y. Kim, J. H. Jang and J. K. Park, RSC $A d v ., 2016,6$, 22424-22435.

19.I. Ahmad, S. B. Khan, T. Kamal and A. M. Asiri, J. Mol. Liq., 2017, 229, 429-435.

20.F. Ali, S. B. Khan, T. Kamal, K. A. Alamry and A. M. Asiri, Sci. Rep., 2018, 8, 6250 .

21.K. M. L. Taylor, J. S. Kim, W. J. Rieter, H. An, W. Lin and W. Lin, J. Am. Chem. Soc., 2008, 130, 2154-2155.

22.L. Ali Shah, Z. Hussain Farooqi, H. Naeem, S. Mujtaba Shah and M. Siddiq, J.Chem.Soc.Pak., 2013, 35, 1522-1529.

23.J. Lü, Y. Yang, J. Gao, H. Duan and C. Lü, Langmuir, 2018, 34, 8205-8214.

24.S. B. Khan, F. Ali, T. Kamal, Y. Anwar, A. M. Asiri and J. Seo, Int. J. Biol. Macromol., 2016, 88, 113-119.

25.F. U. Khan, Asimullah, S. B. Khan, T. Kamal, A. M. Asiri, I. U. Khan and K. Akhtar, Int. J. Biol. Macromol., 2017, 102, 868-877.

26.F. Ali, S. B. Khan, T. Kamal, Y. Anwar, K. A. Alamry and A. M. Asiri,
Chemosphere, 2017, 188, 588-598.

27.S. B. Khan, S. A. Khan, H. M. Marwani, E. M. Bakhsh, Y. Anwar, T. Kamal, A. M. Asiri and K. Akhtar, RSC Adv., 2016, 6, 110077-110090.

28.L. A. Shah, J. Ambreen, I. Bibi, M. Sayed and M. Siddiq, J. Chem. Soc. Pak., 2016, 38, 850-858.

29.M. Ajmal, S. Demirci, M. Siddiq, N. Aktas and N. Sahiner, New J. Chem., 2016, 40, 1485-1496.

30.N. Sahiner, S. Sagbas, M. Sahiner, C. Silan, N. Aktas and M. Turk, Int. J. Biol. Macromol., 2016, 82, 150-159.

31.S. Yildiz, M. Sahiner and N. Sahiner, Eur. Polym. J., 2015, 70, 66-78,.

32.S. A. Khan, S. B. Khan, T. Kamal, A. M. Asiri and K. Akhtar, Recent Pat. Nanotechnol., 2016, 10, 181-188.

33.T. Kamal, S. B. Khan and A. M. Asiri, Cellulose, 2016, 23, 1911-1923.

34.N. Sahiner, H. Ozay, O. Ozay and N. Aktas, Appl. Catal. B Environ., 2010, 101, 137-143.

35.D. Jana, A. Dandapat and G. De, Langmuir, 2010, 26, 12177-12184.

36.A. Leelavathi, T. Bhaskara Rao and T. Pradeep, Nanoscale Res. Lett., 2011, 6, 123.

37.X. W. Wang, K. L. Wu, K. Liu, W. Z. Wang, Y. X. Yue, M. L. Zhao, J. Cheng, J. Ming, X. W. Wei and X. W. Liu, Cryst. Eng. Comm, 2015, 17, 734-739.

38.Y. Mei, Y. Lu, F. Polzer, M. Ballauff and M. Drechsler, Chem. Mater, 2007, 19, 1062-1069.

39.S. H. Wu, C. T. Tseng, Y. S. Lin, C. H. Lin, Y. Hung and C. Y. Mou, J. Mater. Chem., 2011, 21, 789-794.

40.J. Hu, Y. Dong, Z. ur Rahman, Y. Ma, C. Ren and X. Chen, Chem. Eng. J., 2014, 254, 514-523.

41.H. Wu, Z. Liu, X. Wang, B. Zhao, J. Zhang and C. Li, J. Colloid Interface Sci., 2006, 302, 142-148.

Publisher's Note Engineered Science Publisher remains neutral with regard to jurisdictional claims in published maps and institutional affiliations. 